\title{
An Interview with David Ruelle
}

Hans Henrik Rugh (Université Paris-Saclay, Orsay, France

Translation by Rafael Sasportes of the original article titled "Un interview de David Ruelle", published in La Gazette des Mathématiciens, July 2019. The permission of Hans Henrik Rugh, David Ruelle and the Société Mathématique de France is gratefully acknowledged.

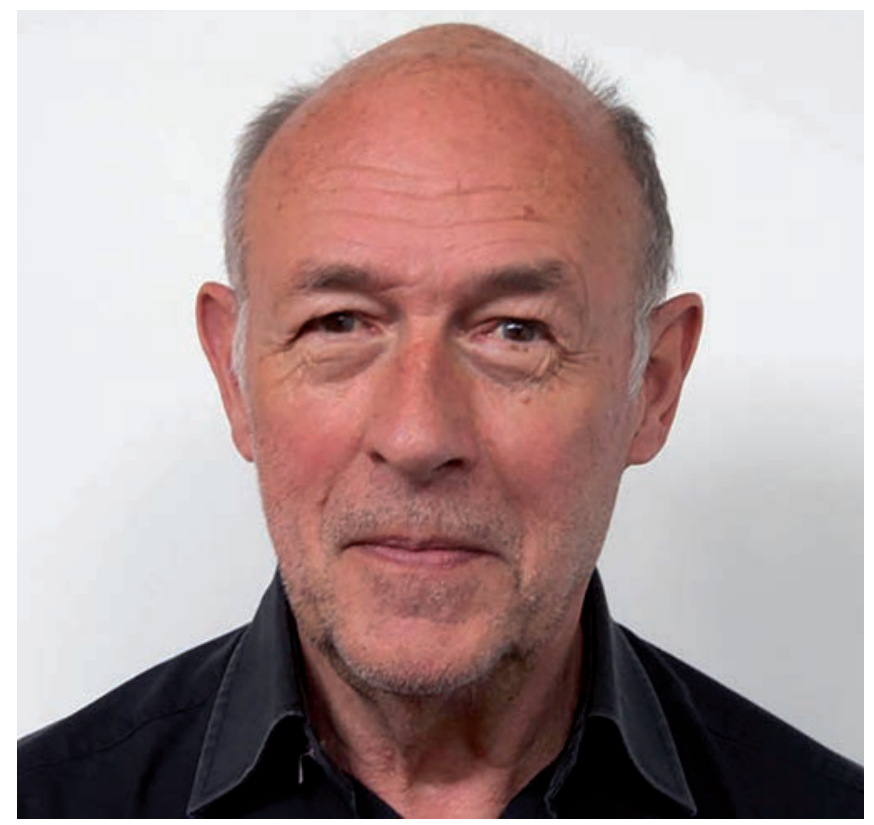

David Ruelle's work covers various fields bordering on physics and mathematics. Some of his best-known results are found in his quantum field theory work on the asymptotic condition (Haag-Ruelle theory), in statistical mechanics in the works which describe "the" natural definition of equilibrium states and Gibbs states for infinite systems (DLR equations: Dobrushin-Lanford-Ruelle), in dynamical systems and turbulence in a proposition with F. Takens on the role of strange attractors to explain turbulence, in differential dynamical systems: SRB states (Sinai-Ruelle-Bowen), the notions of transfer operators and dynamical zeta functions, and also work on out of equilibrium statistical mechanics. He wrote books still considered founding references of their fields: Statistical Mechanics, Rigorous Results (1969) and Thermodynamic Formalism (1978). Among his books for the general public are Chance and Chaos (1991) and The Mathematician's Brain (2007).

Dear David Ruelle, when and how did you become interested in science?

I was very curious from an early age. But while some friends were aces at predicting football results or collected license plate numbers, I was curious about the nature of things. I identified plants using a small scientific book on botany, I did fun (and a little dangerous) chemistry experiments in the basement of my house. I also read parts of the Positive Philosophy Course from Auguste Comte and from the Ethics of Spinoza. I quickly understood that the title Ethica Ordine Geometrico Demonstrata was an illusion: we are a long way away from Euclid's logic. However, I have great personal sympathy for Spinoza, who earned his living by trimming optical lenses and was rejected by his Jewish community for his free thinking. For the rest, I read any scientific and mathematics books that fell into my hands.

At the beginning of your career, how did you judge the scientific environments that you encountered in different countries?

I started my university studies at an engineering school in Mons, the Belgian provincial city where I lived. The courses were, by the way, excellent. Then I continued studying physics and maths at the Free University of Brussels. I studied quantum mechanics with J. Géhéniau, who knew it well, although it was not obvious at the time. I also had time for non-university activities (in particular anti-militarist ones). From Brussels I went to Zürich to work with W. Pauli, who Géhéniau knew. Pauli died shortly after (late 1958), and I became part of a small group who had gathered around Res Jost. I have little enthusiasm for the notions of master and student in science, but I readily admit that the personality of Res Jost was decisive for my beginnings in research (into quantum field theory). Later I changed direction and benefitted a lot from my contacts with other scientists, for example by reading and rereading articles by S. Smale, R.L. Dobrushin and Ia.G. Sinai, and also by interacting with younger colleagues like Oscar Lanford, Jean-Pierre Eckmann and Giovanni Gallavotti. But it's in Zürich that I started a career as a researcher and a life outside of Belgium.

Being a foreigner in the country where you live is a situation well known to many colleagues: you cannot really be politically active and you are exposed to xenophobia by some. I lived as a foreigner in Switzerland, in the United States and in France for a quarter of a century. You get used to it: you are silent and you don't think less of it. This has undoubtedly strengthened my natural tendency to try to understand things and people rather than judging that this is good and this is bad.

At the beginning of my scientific career I saw Niels Bohr, I attended classes by Heisenberg and Pauli and I knew the latter personally. It was the end of a great period for physics. As far as I am concerned, I have become increasingly oriented towards theoretical physics problems, which are mathematical problems with a particular flavour. 
Here I would like to say a few words about the changing atmosphere of scientific research. Quantitatively, there has been a huge increase in the number of researchers within a century. Qualitatively, what was above all a vocation (supported materially by teaching tasks or other sources of income) has become a profession like any other. Instead of individuals seeking to understand the nature of things, there are a host of postdocs seeking a research subject. The financial scope of research has become major, and the role of scientific administrators (often former researchers) has become dominant. The result is a new standard of research: the need to publish in prestigious journals, the obligation to study postdocs applications, etc. Unfortunately, these obligations discourage exceptional scientists, who are often the most original and the best. That said, contemporary research has yielded extraordinary results in many areas.

You have written a few popular science articles and books, often marked by a rather philosophical approach. How do you see the interaction between philosophy and science?

Galileo wrote that the great book of nature was written in mathematical language. It's very well expressed, but if we look at things four centuries later, we see them as a little more complicated. We can say that human mathematics results from the interaction of the human brain with the physical universe in which we live. An axiomatic presentation of mathematics (Zermelo-Fraenkel-Choice) eliminates the physical universe and at the same time the human brain. It should also be noted that understanding the physical universe requires only a part of mathematics based on ZFC. My colleague Giovanni Gallavotti believes that physics can do without the Axiom of Choice (this does not prevent the fact that the inclusion of the Axiom of Choice makes the presentation of mathematics much more natural). In fact, when we work to rewrite the great book of nature in mathematical language, we are led to natural mathematical choices different from those of the usual mathematics based on ZFC. This is not a new thing: the beginnings of geometry (based on the observation of the physical world) imposed the primacy of the real numbers field over other fields.

If we want to understand the nature of things, it seems that we have to start with the study of philosophy. But if we look at the situation more closely, we see that philosophy breaks up in a multitude of doctrines which bring no certainty. The near certainties we have come from maths and science. It should be added that these are quasi-certainties on a human level at a period close to the year 2000, and that the expression of these quasi-certainties is largely based on the use of natural languages such as English and French. It should also be noted that in science and in the practice of mathematics, as in this discussion, the use of natural languages is poorly formalised.

I do not despise philosophy and I have read the dialogues of Plato with pleasure. I am very aware of the evolution of human thought that has led to our current understanding of the nature of things.

To what extent does what we know of the nature of things depend on the human nature of our intelligence? The problem is most affordable in mathematics, which can be formally formulated without resorting to natural languages.

Another question is to know what it is in the personal philosophy of the builders of science that guides them towards their discoveries.

The interaction between mathematics and physics obviously plays an important role in your work. How do you see the structure of mathematics and its use in the description of natural sciences?

Mathematics can be formalised using the ZermeloFraenkel-Choice axioms, which de facto form the basis of current mathematics. We can organise known human results within the framework of the "fundamental structures of analysis" as N. Bourbaki does. But this structuring is far from the ZFC axioms. Other structures use categories, morphisms and functors, etc. Is there a natural structure of mathematics?

Part of the answer could be provided by computers. There are already very reliable formal computer proofs. These computer proofs are logically long, but their accuracy depends on a logical kernel which is a carefully checked short program. If we admit that the basic axioms of mathematics are non-contradictory, formal computer proofs are much more reliable than traditional human proofs. What computers lack is creativity: guessing new results and guessing a way to prove them. Some believe that the creative power of the human brain can never be replaced by "a machine" (a computer). But this belief has no serious scientific basis. In short, it is possible that a natural structure of mathematics could emerge from computer-created mathematics, but that is by no means certain. For now we only know human mathematics.

However, human mathematics is partly guided by our efforts to interpret the physical world around us. My personal research work falls within this framework, that of mathematical physics. As it currently exists, mathematical physics includes quite diverse things, such as string theory, whose relationship with the physical world is not guaranteed. My interests have mainly focused on statistical physics, which is the study of material systems with a large number of particles, such as liquids and gases. It turns out that we can define so-called equilibrium states for these systems, characterised by variables such as temperature, entropy, etc. Ludwig Boltzmann (with Maxwell, Gibbs and others) understood what an equilibrium state is: it is a specific probability measure over a space with large dimension. This measure corresponds to a given interaction between the particles of the system.

We will not try to summarise the theory of equilibrium states (statistical mechanics of equilibrium) here. Let's just say it is a mathematically difficult but natural theory, it introduces important mathematical concepts like entropy, and there are still poorly understood phe- 
nomena like phase transitions. An important notion that I have studied (with R.L. Dobrushin and O. Lanford) is that of Gibbs state.

Let's move on to a mathematical problem unrelated to the statistical mechanics of equilibrium: hyperbolic differentiable dynamical systems. For these systems, Ia.G. Sinai has constructed what he calls Markov partitions which allow an application of the methods of statistical equilibrium mechanics. It turns out that Gibbs states are a wonderful tool for an in-depth study of the theory of (uniformly) hyperbolic systems. Hyperbolic dynamical systems appear in the study of hydrodynamic turbulence (D. Ruelle and F. Takens). We thus see that there is a purely mathematical link (via Gibbs states) between two very different physical problems: the statistical mechanics of equilibrium and hydrodynamic turbulence. How is that possible?

In my opinion, the study of mathematical physics leads to the introduction of fruitful mathematical concepts, like entropy or Gibbs states. These concepts would be much more difficult to find in a Bourbaki-style mathematical approach based on the analysis of structures. There is here an element to answering the question: is there a natural structure of mathematics?

In the area of dynamical systems, part of the terminology has been imported from statistical mechanics. What is the explanation for this?

I had to work on both sides of the border between physics and mathematics. I will not list these works here. But it turns out that I contributed to the terminology of differentiable dynamical systems. For instance, I introduced the term "thermodynamic formalism" - this is the title of a book I published in 1978. I also introduced the term "pressure" for a function that appears in ergodic theory; frankly the proper term in statistical mechanics should be "free energy", but the word pressure seemed more acceptable to mathematicians. I should also mention the expression "strange attractor", which has been very successful and seems to have appeared for the first time in an article published by Floris Takens and myself in 1971. The intention of the article was to clarify a small mathematical point concerning hydrodynamic turbulence. I thought the article would go unnoticed and be immediately forgotten, instead of which it has been cited to date 3874 times. Vanitas vanitatum!

One question I have worked on is that of SRB states for differentiable dynamical systems. The initials SRB correspond to Sinai, Ruelle, and Bowen, but one should also add F. Ledrappier, J.M. Strelcyn, L.-S. Young and a few others. My interaction with Sinai and Bowen was a great source of job satisfaction for me: everyone just wanted to understand a problem, not to show that he was superior to his colleagues. Yasha Sinai was a great force in Russian mathematics. As for Rufus Bowen, who died at 31 , his clarity of mind was extraordinary, and a difficult problem suddenly became simple when he explained it. I also had a lot of fun at the IHÉS interacting with Viviane Baladi and Hans Henrik Rugh on issues of differentiable dynamics.
You have been a member of several academies for a good number of years. What do you think of the role of academies and their function of scientific assessment?

For science to progress, the value of scientific work must be constantly assessed. This assessment takes several forms: selection of articles in peer-reviewed scientific journals, scientific honours, etc. Any form of assessment has an element of power, and power corrupts. It is therefore desirable that this power is not absolute, and that there is a balance of these assessment powers. At the moment, it seems to me that there is a problem in the growing role of professional scientific administrators, and also in the biased or incompetent attribution of certain great scientific prizes. Any power of scientific assessment can be criticised, it is the case of scientific honours like the election to an academy. As much as the way academies work deserves to be discussed, the abolition of these would only encourage other instances of power, without this benefitting science.

Personally, I am a member of several academies, including the Paris Academy of Sciences, where I play a modest role. It is clear in my mind that slightly different circumstances could have led to me not becoming an academy member. That said, the greatest benefit I have derived from the academy is to have rubbed shoulders with a generation of great mathematicians: H. Cartan, J. Leray, J.-P. Serre, L. Schwartz, J. Dieudonné, R. Thom, J. Tits, G. Choquet and others.

Who are the most memorable scientific minds in your career?

During my military service as a private in the Belgian army, I was in a room with two illiterates: one had never learned to read, the other had forgotten how to. It was an intellectually disadvantaged environment. However, I met other privates there who were remarkable men. In a military environment, one of whose mission was to break personalities, these men, either by courage, trickery or cheating, did not break. In my scientific career too, I have met remarkable individuals. Often when I have to make a decision, I think of this or that person, and I wonder what he or she would have done in this situation. Important scientists are not necessarily remarkable people in my opinion. Many are specialists with limited intellectual ambitions, such as becoming a head mathematician. Grothendieck, on the other hand, said: I am a generalist, not a specialist.

Alexandre Grothendieck had an extraordinary personality. He wrote a praise for incest. His relationships with the women who shared his life could be extremely tense. He was firmly anti-militarist and had anarchist tastes. Mathematics played a central role in his life at certain times, meditation and religion at other times. I was around Grothendieck for several years and I was fascinated by the way he worked, including his changes in orientation. He faced general human problems in an original way. You can follow or not follow his choices, but when I have to make a decision I often wonder what Grothendieck would have done in my place. 
As rich as Grothendieck's life seems to me, including its contradictions, the corrected version that is often made of it now seems false and lacklustre. Grothendieck would be a chief mathematician with politically correct ideas (by today's standards) and a few errors that must be excused because he was a genius.

Remarkable scientific minds have very diverse intellectual personalities, and it is this diversity that allows them new approaches to scientific problems. But it seems to me that remarkable scientific minds are often guided by the search for a general view of the nature of things and the universe in which we live. This was certainly the case with Newton, who, in addition to his contributions to mathematics and physics, devoted great efforts to alchemy and theology. Spinoza, Newton or Grothendieck's efforts to understand the nature of things have sometimes led to remarkable discoveries, sometimes not. During conversations with Murray Gell-Mann I was impressed by his knowledge of languages and biology, here was someone who sought a general understanding of the nature of things, and not just of one area of physics. These examples show that trying to guide research by administrative standards can only impoverish our knowledge of the world.

My curiosity about the nature of things has never been limited to my professional scientific work. Among other things, I have spent time hiking, alone or with my wife, in remote corners of Mexico, Bolivia, or elsewhere. In the scientific field I have had the satisfaction of gaining a certain understanding of scientific fields such as the statistical mechanics of equilibrium or the theory of chaos, or of clarifying small questions like the lemma of Asano-Ruelle (concerning the zeros of complex quadratic polynomials with two variables). I have admired the mathematical way in which minds like those of Bowen and Sinai work. I have also been able to see remarkable spirits acting in ordinary life, such as Mark Kac full of humour, Joel Lebowitz always generous, or J. Robert Oppenheimer colder and for whom I had less sympathy. But sympathy or not, the different ways in which great scientific minds work have always fascinated me.

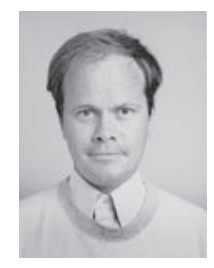

Hans H. Rugh wrote his PhD thesis in Copenhagen, Denmark, under the supervision of Predrag Cvitanović. He has since held full-time positions in Warwick, England, as well as in Cergy-Pontoise, France. At present he is professor at the University of Paris-Saclay, at the Mathematics Institute at Orsay. 